\title{
Comparison between Adductor Canal Nerve Block and Femoral Nerve Block in Postoperative Pain Management after Arthroscopic Knee Surgeries
}

Fawzy Abbas Badawy, Mohsen Mohammed El Sayed*, Khaled Abdelfattah Mohamed Abdelfattah

Department of Anesthesia, ICU \& Pain Management, Faculty of Medicine, Sohag University

*Corresponding author: Mohsen Mohammed El Sayed, Mobile: (+20)01144336881,

E-Mail: mohsentakhder@gmail.com

\begin{abstract}
Background: Knee arthroscopy is one of the surgeries of lower limbs, which are associated with postoperative pain and the patients generally require a significant amount of opioid-based analgesics after such procedures. Treatment of the postoperative pains after surgical operations of lower limbs is of special importance. Inadequate treatment of these pains results in the reduction of patient's activities and related complications.

Objective: To evaluate and compare the efficacy, duration of sensory block, duration of postoperative analgesia and complication between adductor canal block (ACB) and femoral nerve block (FNB).

Patients and methods: This randomized controlled study was conducted at Sohag University Hospital. Fourty patients undergoing elective arthroscopic knee surgeries under general anesthesia were included in the study after giving written and informed consents.

Results: In our study, there was a statistically significant difference between the studied groups regarding onset and duration of sensory block peak (both were higher in ACB group). Regarding VAS, there was a statistically non-significant difference between the studied groups preoperatively and at 3,6 and 12 hours postoperatively, while significant difference was detected between them at 24 hours postoperatively (higher VAS score in ACB group at 24 hours). On assessing change overtime in each group, the difference was statistically significant (significant fluctuation over time). But, there was statistically non significant difference between the studied groups regarding modified Ramsey before or one hour after nerve block.
\end{abstract}

Conclusion: ACB provided comparable analgesic efficacy and facilitated earlier mobilization by sparing quadriceps strength compared to FNB.

Keywords: Adductor canal nerve block, Femoral nerve block, Pain management, Knee surgeries.

\section{INTRODUCTION}

Knee arthroscopy is a common orthopedic procedure worldwide. Despite its minimally invasive nature compared to the traditional knee surgery, post arthroscopic pain may be severe and the patients generally require a significant amount of opioid-based analgesics after such procedure ${ }^{(\mathbf{1})}$.

Peripheral nerve blocks offer effective analgesia and decrease the need for opioids, thereby reducing the complications associated with the use of this class of drugs (2). Moreover, postoperative pain relief is an important factor in the early ambulation and rehabilitation of patients after knee surgery ${ }^{(3)}$.

The lumbar plexus consists of sensory and motor nerves, which innervates visceral organs in the pelvis, anterior, anterolateral dermatomes of the thigh and the medial dermatome of the lower leg, as well as provides motor control for the quadriceps femoris muscle ${ }^{(4)}$.

The femoral nerve is one of the most important nerves of the anterior division group of nerves in the lumbar plexus, which mainly supplies the sensation for the anterior and medial parts of the lower extremities ${ }^{(5)}$. Given excellent pain relief and the opioid sparing effect, femoral nerve block (FNB) is commonly used as an analgesic modality and is considered the standard PNB in patients undergoing knee arthroscopy. However, FNB is followed by a significant decrease in quadriceps muscle strength, resulting in delayed mobilization, which is associated with the potential risk of falling ${ }^{(6)}$.

Adductor canal block (ACB) is a relatively an alternative for post-knee surgeries pain management. Regional anesthesia is deposited within an adductor canal that can be easily visualized at the middle third of the thigh with use of ultrasonography ${ }^{(7)}$. As the length of stay in hospital has been shortened by the performance of knee arthroscopy on an outpatient basis, a potent analgesia that preserves motor strength during early rehabilitation is becoming increasingly accepted as an essential part of the current perioperative protocol following knee arthroscopy. A growing body of evidence supports the use of an adductor canal block (ACB) that offers almost pure sensory block with minimal motor involvement as part of a multimodal approach to pain control after knee arthroscopy ${ }^{(8)}$.

As the emphasis has been on faster recovery during the early postoperative period, recent trends in pain management protocols following knee arthroscopy have shifted toward effective analgesia with limited motor involvement. FNB is a commonly 
used modality as part of knee arthroscopy pain control regimens and is considered the gold standard for postoperative analgesia after knee arthroscopy. However, it may reduce quadriceps strength, which is essential for early mobilization and is associated with an increased risk of postoperative falls. Thus, ACB has emerged as a reasonable alternative to FNB that produces a predominantly sensory block with greater quadriceps strength preservation ${ }^{(9)}$.

\section{AIM OF THE WORK}

To evaluate and compare the efficacy, duration of sensory block, duration of postoperative analgesia and complication between adductor canal block and femoral nerve block.

\section{PATIENTS AND METHODS}

This randomized controlled study was done at Sohag University Hospital. Fourty patients undergoing elective arthroscopic knee surgeries under general anesthesia (GA) criteria were included in the study after giving written and informed consents.

Inclusion criteria: American Society of Anesthesiology (ASA) physical status I and II. Age 18-60 years. Either male or female sex. Patients who performed knee arthroscopic surgeries.

Exclusion criteria: ASA class III and IV. History of coagulopathy or any blood diseases. Receiving any anticoagulants or antiplatelet therapy. Patients with history of allergic reaction to the study drugs. Refuse local anesthetics. Patients complaining from cardiac, hepatic, renal and chest diseases or diabetes. Patients with advanced uncontrolled diabetes with glycated haemoglobin (HbA1c) percentage $>7 \%$. Obesity (body mass index of $>35$ ). Alcoholism. Infection at the site of injection.

\section{Ethical approval and patients' consent:}

An approval of the study was obtained from Sohag University academic and ethical committee. Every patient signed an informed written consent for acceptance of the operation.

\section{Anesthetic plan:}

Preoperative assessment was done for all patients the day before surgery. All patients were premedicated in the holding room with IV administration of midazolam hydrochloride $(0.05$ $\mathrm{mg} / \mathrm{kg}$ ) half an hour before the induction of GA. Following their transfer to the operating room, general anesthesia was induced with IV administration of propofol $(2 \mathrm{mg} / \mathrm{kg})$ and fentanyl $(3 \mu \mathrm{g} / \mathrm{kg})$, then endotracheal intubation was done with cisatracurium $(0.2 \mathrm{mg} / \mathrm{kg})$ for muscle paralysis. Anaesthesia was maintained with inhalation of isoflurane. Muscle paralysis was maintained with intermittent boluses of cisatracurium. Then, the patients were transferred to recovery room after extubation. All patients were blocked under US guidance after surgery in the recovery room. After positioning of the lower extremity in slight external rotation and $30^{\circ}$ knee flexion, the thigh was prepped with $70 \%$ isopropyl alcohol.

In the FNB group, the femoral nerve was blocked at level of inguinal ligament. In brief, the femoral artery was identified below inguinal ligament under ultrasound guidance with a linear probe. The femoral nerve was detected adjacent to the artery using subsequently $12 \mathrm{~mL}$ of bupivacaine $0.125 \%$ was injected along the nerve sheet after negative aspiration.

In the adductor canal block (ACB) group, a linear ultrasound probe was placed in inner thigh. The femoral artery is located at one third of the inner thigh under the sartorius muscle. The ultrasound probe was moved to the caudal direction until technically the superficial femoral artery or just femoral artery was identified. After identifying femoral canal, adductor hiatus of the saphenous nerve was visualized as hyperechoic structure superficial and lateral to femoral artery then $12 \mathrm{~mL}$ of bupivacaine $0.125 \%$ was injected to the nerve sheet using nerve stimulator to elicit dysesthesias in the distribution of the saphenous nerve.

\section{Monitoring:}

After the block, the severity of pain was measured using visual analogue scale (VAS) by a member of the study team, who has no prior knowledge of the type of the nerve block, at 0 (in the recovery room), 3, 6, 12 and 24 hours after nerve block. VAS of pain is an ordinal data ranging from 0 for no pain to 10 for the worst imaginable pain on a 10 cm scale.

Anxiety and apprehension were evaluated based on modified Ramsay sedation-agitation scale (SAS) from 0 to 5 where $0=$ Unresponsive, $1=$ drowsy and sleepy, $2=$ calm and cooperative patient, $3=$ apprehension, $4=$ agitation and $5=$ excessive agitation and combative behavior immediately after recovery and after an hour ${ }^{(\mathbf{1 0})}$. The level of anxiety and agitation was assessed before and 1 hour after performing nerve block in all patients. Patient satisfaction with the quality of analgesia was assessed using a Likert-based questionnaire given to the patients where a score of 1 indicated strongly dissatisfied and score of 5 indicated strongly satisfied. To standardise supplemental pain control in the two groups, parenteral 6 acetaminophen $1000 \mathrm{mg}$ was administered every 6 hours in each group and in the case of no improvement; additional $1000 \mathrm{mg}$ was administered up to a maximum dose of 6 grams in 24 hours exceeding MDD in 24 hours.

The extent of motor block was assessed using a modification made on the Bromage scale as follows: 0: if there is no residual motor weakness in leg muscles, 1 $=$ if the patient is unable to flex the hip joint against gravity, $2=$ if the patient is unable to extend the knee against gravity and $3=$ if the patient is unable to flex the hip joint and extend the knee joint against gravity. The presence and the degree of motor blockade was assessed at the same time points as we performed for the presence of pain. 


\section{Statistical analysis}

Recorded data were analyzed using the statistical package for social sciences, version 20.0 (SPSS Inc., Chicago, Illinois, USA). Quantitative data were expressed as mean \pm standard deviation (SD). Qualitative data were expressed as frequency and percentage. Independentsamples t-test of significance was used when comparing between two means. Chi-square $\left(\mathrm{x}^{2}\right)$ test of significance was used in order to compare proportions between two qualitative parameters. The confidence interval was set to $95 \%$ and the margin of error accepted was set to $5 \%$. The p-value was considered significant as the following: $\mathrm{P}$-value $\leq 0.05$ was considered significant. P-value $<0.001$ was considered as highly significant. P-value $>0.05$ was considered insignificant.

\section{RESULTS}

Table (1): Comparison between the studied groups regarding demographic characteristics

\begin{tabular}{|l|c|c|c|c|}
\hline \multirow{2}{*}{$\begin{array}{c}\text { Demographic } \\
\text { characteristics }\end{array}$} & \multicolumn{2}{|c|}{ Groups } & \multicolumn{2}{c|}{ Test } \\
\cline { 2 - 4 } & Adductor canal block group & $\begin{array}{c}\text { Femoral nerve } \\
\text { block group }\end{array}$ & \multirow{2}{*}{$\chi^{2 / t}$} & p \\
\cline { 2 - 4 } & $\mathbf{N = 2 0 ( \% )}$ & $\mathbf{N = 2 0 ( \% )}$ & \\
\hline $\begin{array}{l}\text { Gender: } \\
\text { Male }\end{array}$ & $\begin{array}{c}15(75) \\
\text { Female }\end{array}$ & $\begin{array}{c}13(65) \\
7(35)\end{array}$ & 0.476 & 0.49 \\
\hline $\begin{array}{l}\text { Age (years) } \\
\text { Mean } \pm \text { SD }\end{array}$ & $29.35 \pm 5.47$ & $30.8 \pm 8.75$ & -0.629 & 0.534 \\
\hline
\end{tabular}

$\chi^{2}$ Chi square test

$\mathrm{t}$ Independent sample $\mathrm{t}$ test

There was a statistically non-significant difference between the studied groups regarding gender or age (Table 1).

Table (2): Comparison between the studied groups regarding ASA and past history

\begin{tabular}{|c|c|c|c|c|}
\hline \multirow[t]{3}{*}{ Clinical data } & \multicolumn{2}{|c|}{ Groups } & \multicolumn{2}{|c|}{ Test } \\
\hline & $\begin{array}{l}\text { Adductor canal } \\
\text { block group }\end{array}$ & $\begin{array}{c}\text { Femoral nerve block } \\
\text { group }\end{array}$ & $\chi^{2}$ & $\mathbf{p}$ \\
\hline & $\mathrm{N}=20(\%)$ & $\mathbf{N}=\mathbf{2 0}(\%)$ & & \\
\hline $\begin{array}{l}\text { Diagnosis: } \\
\text { ACL injury } \\
\text { Meniscal injury } \\
\text { Meniscal tear } \\
\text { PCL injury }\end{array}$ & $\begin{array}{c}9(45) \\
0(0) \\
7(35) \\
3(15)\end{array}$ & $\begin{array}{c}9(45) \\
8(40) \\
0(0) \\
3(15)\end{array}$ & 0.983 & 0.322 \\
\hline $\begin{array}{l}\text { Operation } \\
\text { Knee arthroscopy }\end{array}$ & $20(100)$ & $20(100)$ & 0 & $>0.999$ \\
\hline
\end{tabular}

$\chi^{2}$ Chi square test

There was statistically non-significant difference between the studied groups regarding their diagnosis or type of operation. Forty five percent within both groups had anterior cruciate ligament (ACL) injury. No patient within ACB group had meniscal injury versus 8 patients (40\%) within FNB group. 7 patients (35\%) in ACB had meniscal tear versus $0 \%$ of those within FNB group. In addition, there was 3 patients $(15 \%)$ in each group had posterior cruciate ligament injury (PCL) (Table 2).

Table (3): Comparison between the studied groups regarding VAS score over time

\begin{tabular}{|c|c|c|c|c|c|c|}
\hline \multirow[t]{2}{*}{ VAS } & \multicolumn{2}{|c|}{ dductor canal block group $(\mathrm{n}=20)$} & \multicolumn{2}{|c|}{$\begin{array}{c}\text { Femoral nerve block group } \\
(\mathrm{n}=20)\end{array}$} & \multirow[t]{2}{*}{$\mathbf{t}$} & \multirow[t]{2}{*}{$\mathbf{p}$} \\
\hline & Mean \pm SD & Range & Mean \pm SD & Range & & \\
\hline Preoperative & $7.4 \pm 0.75$ & $6-9$ & $7.65 \pm 0.67$ & $7-9$ & -1.108 & .275 \\
\hline 3 hours postop. & $3 \pm 0.65$ & $2-4$ & $3.15 \pm 1.14$ & $2-7$ & 0.153 & .611 \\
\hline 6 hours postop. & $4.1 \pm 0.79$ & $2-5$ & $3.85 \pm 0.76$ & $3-6$ & 1.08 & .287 \\
\hline 12 hours postop. & $5.3 \pm 0.57$ & $4-6$ & $5.05 \pm 0.6$ & $4-6$ & 1.344 & .187 \\
\hline 24 hours postop. & $5.9 \pm 0.72$ & $5-7$ & $5.4 \pm 0.6$ & $4-6$ & 2.392 & $022 *$ \\
\hline $\mathbf{P}_{2}$ & \multicolumn{2}{|c|}{$<0.001 * *$} & \multicolumn{2}{|c|}{$<0.001 * *$} & & \\
\hline
\end{tabular}

$\mathrm{t}$ Independent sample $\mathrm{t}$ test

$\mathrm{P}_{2} \mathrm{p}$ for repeated measure ANOVA

$* \mathrm{p}<0.05$ is statistically significant $\quad * * \mathrm{P} \leq 0.001$ is statistically highly significant

There was a statistically non-significant difference between the studied groups regarding VAS preoperatively and at 3,6 and 12 hours postoperative while significant difference was detected between them at 24 hours postoperatively (higher VAS score in ACB group at 24 hours). On assessing change overtime in each group, the difference was statistically significant (significant fluctuation over time) as shown in table (3). 
Table (4): Comparison between the studied groups regarding modified Ramsey score before and one hour after nerve block

\begin{tabular}{|l|c|c|c|c|c|c|}
\hline \multirow{2}{*}{$\begin{array}{l}\text { Modified Ramsey } \\
\text { sedation analgesia score }\end{array}$} & \multicolumn{2}{|c|}{$\begin{array}{c}\text { Adductor canal } \\
\text { block group }\end{array}$} & \multicolumn{2}{|c|}{$\begin{array}{c}\text { Femoral nerve } \\
\text { block group }\end{array}$} & \multicolumn{2}{c|}{ Test } \\
\cline { 2 - 7 } & $\mathbf{N = 2 0}$ & $\mathbf{0}$ & $\mathbf{N = 2 0}$ & $\mathbf{2 0}$ & $\chi^{\mathbf{2}}$ & $\mathrm{p}$ \\
\hline $\begin{array}{l}\text { before nerve block: } \\
\text { Drowsy and sleepy }\end{array}$ & 20 & 100 & 20 & 100 & 0 & $>0.999$ \\
\hline $\begin{array}{l}\text { 1 hour postop } \\
\text { Calm and cooperative }\end{array}$ & 20 & 100 & 20 & 100 & 0 & $>0.999$ \\
\hline
\end{tabular}

$\chi^{2}$ Chi square test

There was statistically non-significant difference between the studied groups regarding modified Ramsey before or one hour after nerve block (Table 4).

Table (5): Comparison between the studied groups regarding likert score for patient satisfaction with analgesia within 24 hour

\begin{tabular}{|l|c|c|c|c|c|c|}
\hline $\begin{array}{l}\text { Patient satisfaction } \\
\text { with anesthesia }\end{array}$ & \multicolumn{2}{|c|}{$\begin{array}{c}\text { Adductor canal } \\
\text { block group }\end{array}$} & \multicolumn{2}{|c|}{$\begin{array}{c}\text { Femoral nerve } \\
\text { block group }\end{array}$} & Test \\
\cline { 2 - 7 } & $\mathbf{N = 2 0}$ & $\mathbf{\%}$ & $\mathbf{N = 2 0}$ & $\mathbf{\%}$ & $\chi^{\mathbf{2}}$ & $\mathrm{p}$ \\
\hline Strongly dissatisfied & 0 & 0 & 0 & 0 & & \\
Somewhat dissatisfied & 0 & 0 & 0 & 0 & 1.08 & 0.298 \\
Neutral & & 35 & 6 & 30 & & \\
Somewhat satisfied & 7 & 25 & 1 & 5 & & \\
Strongly satisfied & 5 & 40 & 13 & 65 & & \\
& 8 & & & & & \\
\hline
\end{tabular}

$\chi^{2}$ Chi square test

There was statistically non-significant difference between the studied groups regarding patient satisfaction with anesthesia (Table 5).

Table (6): Comparison between the studied groups regarding Bromage score over time

\begin{tabular}{|c|c|c|c|c|}
\hline Bromage scale in lower limbs & $\begin{array}{c}\begin{array}{c}\text { Adductor canal } \\
\text { block group }\end{array} \\
\mathbf{N}(\%)\end{array}$ & $\begin{array}{c}\begin{array}{c}\text { Femoral nerve } \\
\text { block group }\end{array} \\
\mathrm{N}(\%)\end{array}$ & $\chi^{2}$ & $\mathbf{P}$ \\
\hline $\begin{array}{l}\text { Preoperatively: } \\
\text { No motor weakness }\end{array}$ & $20(100)$ & $20(100)$ & 0 & $>0.999$ \\
\hline $\begin{array}{l}\text { At } 3 \text { hours: } \\
\text { No motor weakness } \\
\text { Unable to flex against gravity } \\
\text { Unable to extend against gravity }\end{array}$ & $\begin{array}{l}10(50) \\
5(25) \\
5(25)\end{array}$ & $\begin{array}{l}5(25) \\
5(25) \\
10(50)\end{array}$ & 3.333 & 0.189 \\
\hline $\begin{array}{l}\text { At } 6 \text { hours: } \\
\text { No motor weakness } \\
\text { Unable to flex against gravity } \\
\text { Unable to extend against gravity }\end{array}$ & $\begin{array}{l}15(75) \\
4(20) \\
1(5)\end{array}$ & $\begin{array}{l}10(50) \\
2(10) \\
8(40)\end{array}$ & 7.111 & $0.029 *$ \\
\hline $\begin{array}{l}\text { At } 12 \text { hours: } \\
\text { No motor weakness } \\
\text { Unable to flex against gravity }\end{array}$ & $\begin{array}{c}20(100) \\
0(0)\end{array}$ & $\begin{array}{l}15(75) \\
5(25)\end{array}$ & Fisher & $0.047 *$ \\
\hline $\begin{array}{l}\text { At } 24 \text { hour: } \\
\text { No motor weakness }\end{array}$ & $20(100)$ & $20(100)$ & 0 & $>0.999$ \\
\hline $\mathbf{p}$ & $<0.001^{* *}$ & $<0.001 * *$ & & \\
\hline
\end{tabular}

$* \mathrm{p}<0.05$ is statistically significant $* * \mathrm{P} \leq 0.001$ is statistically highly significant

There was statistically non-significant difference between the studied groups regarding bromage score baseline, 3 and 24 hours postoperatively. There was statistically significant difference between the studied groups regarding bromage score at 6 and 12 hours postoperatively (gradual improvement in score among patients with ACB until all patients had no motor weakness on 12 hours. This improvement was present in FNB group yet with lesser percentage to reach $75 \%$ at 12 hours. There was statistically significant change in bromage score between the studied groups over time (Table 6). 


\section{DISCUSSION}

In this study, we observed that there were statistically non-significant differences between the studied groups regarding gender and age. This is in agreement with Rahimzadeh $\boldsymbol{e t}$ al. ${ }^{(11)}$ who compared the analgesic effects of femoral nerve block (FNB) and adductor canal block (ACB) after arthroscopic knee surgery. An equal number of 92 patients aged from 18 to 60 years were assigned to either the FNB or the ACB group ( $\mathrm{N}=46$ in each group). The average age of the subjects was $36.4 \pm 15.5$ years. There was no difference in age and gender.

We observed that there was statistically nonsignificant difference between the studied groups regarding diagnosis or type of operation. Forty five percent within both groups had ACL injury. No patient within ACB group had meniscal injury versus 8 patients (40\%) within FNB group. 7 patients $(35 \%)$ in ACB had meniscal tear versus $0 \%$ of those within FNB group. In addition, there was 3 patients (15\%) in each group had posterior cruciate ligament injury (PCL). Rahimzadeh et al. (11) found no difference between the two groups involving either anterior or posterior cruciate ligaments.

In our study, there was a statistically significant difference between the studied groups regarding onset and duration of sensory block peak (both were higher in ACB group). Regarding VAS, there was statistically non-significant difference between the studied groups preoperatively and at 3,6 and 12 hours postoperatively, while significant difference was detected between them at 24 hours postoperatively (higher VAS score in ACB group at 24 hours). On assessing change over time in each group, the difference was statistically significant (significant fluctuation over time). Nevertheless, there was statistically non-significant difference between the studied groups regarding modified Ramsey before or one hour after nerve block. Rahimzadeh et al. (11) found that VAS scores prior to the placement of block were similar among the patients assigned to either group. Within 3 hours after placement of the nerve block, patients in the FNB group reported higher quality of pain relief compared to those in the ACB group. This significant difference in the VAS pain score lasted for 12 hours and disappeared thereafter, as they were similar at the 24-hour time point. Patients within both groups were relatively comfortable at the 24-hour time point. There were no differences in the VAS pain score among the patients in either group according to the involvement of anterior or posterior cruciate ligaments. Prior to the placement of nerve block, VAS pain scores were not different in patients with no ligament involvement, from patients with involvement of anterior and posterior ligaments. In Kim et al. ${ }^{(12)}$ study found that the ACB group was not inferior to the FNB group at postoperative pain management. At 24 and $48 \mathrm{~h}$, there were no statistically significant difference between groups. The upper limits of the CIs at all time measurements were less than the delta of 1.6, suggesting that ACB was not inferior to FNB with regard to the pain scores at rest throughout the first $48 \mathrm{~h}$. In Memtsoudis et al. (2) found that patients had similar overall pain scores at rest and during exercise when comparing blocks. This is in line with previous reports supporting the effectiveness of ACB in knee arthroplasty and arthroscopy patients.

In our study, there was statistically nonsignificant difference between the studied groups regarding patient satisfaction with anesthesia. In a

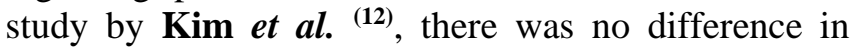
patient satisfaction at 8 hours and 24 hours post anaesthesia between the ACB and the FNB groups. A study by Memtsoudis et al. (2) reported similar findings regarding patient satisfaction. The level of patient satisfaction was directly related to the quality of pain control in all of these studies. Rahimzadeh $\boldsymbol{e t}$ al. (11) found that following performing the nerve block, a significant majority of the patients in both groups $(70.7 \%)$ were cooperative and calm. The percentage of calm and cooperative patients was significantly higher in the FNB group compared to the ACB group. The degree of patient satisfaction with the quality of analgesia for the first 24 hours after surgery was significantly higher among the patients who received FNB compared to those in the ACB group, as $95.7 \%$ of the patients were strongly satisfied with the level of pain control.

Regarding Bromage score, there was statistically non-significant difference between the studied groups at baseline, 3 and 24 hours postoperatively. There was statistically significant difference between the studied groups at 6 and 12 hours postoperatively (gradual improvement in score among patients with ACB, till all patients had no motor weakness on 12 hours, while it was present in FNB group yet with lesser percentage to reach $75 \%$ at 12 hours). On assessing change overtime in each group, there was statistically significant change in Bromage score between the studied groups over time. Rahimzadeh et al. ${ }^{(11)}$ documented that following the deposition of the bupivacaine solution at the block site, significant weakness of quadriceps femoris was observed in an equal number of patients in either group, as 30 patients in each group were unable to extend their knees against the gravity. Weakness of quadriceps femoris more commonly manifested as inability to extend the knee rather than inability to flex the hip joint against the gravity. No patient at any time manifested difficulty in both flexing the hip and extending the knee joints simultaneously. Over $95 \%$ of the patients in both groups were free of motor weakness by 12 hours after the placement of nerve 
block. There was no difference between the FNB and ACB groups in the development of muscle weakness at any time point.

In our study, there was statistically nonsignificant difference between both groups regarding need for analgesia at 24 hours or number of needed doses. Both groups needed analgesia at 24 hours and received two grams for each patient. In another study by Kim et al. (12), no significant difference in supplemental analgesia was observed within 24 and 48 hours after general anaesthesia between the ACB and the FNB groups. Rahimzadeh et al. ${ }^{(11)}$ found that adequate analgesia was also evident in the FNB group as the patients in this group received $4.090 \pm 354 \mathrm{mg}$ of acetaminophen within 24 hours after surgery compared to those in the ACB group who received $5.040 \pm 1.010 \mathrm{mg}$ of the same medication.

Our findings indicated that ACB is one of the most useful analgesic modalities in contemporary perioperative management protocols that focus on rapid recovery after knee surgery. ACB can be performed easily with recently introduced high quality, portable ultrasound technology during surgeries around the knee joint, with high success rates. In addition, it provided excellent pain relief around the knee joint compared to placebo and preserved motor strength with minimal differences from baseline. Wiesmann et al. (13) showed that ACB offered satisfactory analgesic effects with well-preserved mobilization ability in patients who had undergone arthroscopic surgery or knee arthroscopy, suggesting that ACB should be taken into account as part of a contemporary multimodal approach to pain control after knee arthroscopy. ACB, which offers almost pure sensory blockade, seems to be a reasonable alternative to FNB that leads to substantial reduction in quadriceps muscle strength, as part of a current knee arthroscopy pain control protocol. Elkassabany et al. (14) showed comparable analgesic efficacy in terms of pain level and opioid consumption between ACB and FNB. In addition, Burckett-St Laurant et al. (15) documented superior quadriceps strength and mobilization ability during the first 24 hours after knee arthroscopy for ACB compared to FNB. Moreover, $\mathrm{ACB}$ catheters required fewer provider interventions per patient, thus decreasing the workload compared to FNB catheters. We suggested that ACB is a more appropriate analgesic modality than FNB in patients undergoing current multimodal perioperative protocols after knee arthroscopy. However, superior functional recovery in ACB group was limited to the 24-48-hour period after knee arthroscopy. In addition, although ACB offered comparable pain relief with preserved motor strength, patient satisfaction did not differ. Moreover, there was no evidence that ACB reduced the risk for postoperative falls, which may be a fatal complication of FNB or length of stay compared to FNB.

A limited number of studies have examined the anatomy and infiltration technique of ACB. In addition, studies comparing ACB to FNB in terms of analgesic efficacy and functional recovery in patients undergoing knee arthroscopy remain limited. Ludwingson et al. ${ }^{(16)}$ found that single injection ACB offered similar pain control and earlier discharge compared to continuous FNB in patients undergoing total knee arthroplasty. Li et al. ${ }^{(17)}$ found that after total knee arthroplasty, ACB provided better ambulation ability and faster functional recovery along with a better pain control at rest compared to FNB. In a study by Memtsoudis et al. ${ }^{(2)}$, motor strength as assessed manually, using a dynamometer (Lafayette Manual Muscle Test System) was not significantly different between ACB and FNB. Machi et al. ${ }^{(18)}$ also found that continuous ACB compared to continuous FNB decreased the time until adequate mobilization but not overall time to discharge readiness. Decision about perfroming continuous $\mathrm{ACB}$ is based on the anesthesiologist's judgment, required duration of analgesia and the use of adjunct pain medications. Rahimzadeh et al. ${ }^{(11)}$ demonstrated that patients with FNB had denser analgesia after arthroscopic knee surgery and had less analgesic requirement compared to ACB. Greater satisfaction scores also reflected superior analgesia in patients receiving FNB.

\section{CONCLUSION}

ACB provides comparable analgesic efficacy and facilitates earlier mobilization by sparing quadriceps strength compared to FNB. Based on current trends in perioperative protocols toward early rehabilitation following knee arthroscopy, ACB may be a reasonable alternative to FNB as part of a contemporary multimodal pain management protocol after knee arthroscopy.

We recommend placing FNB after arthroscopic surgery of the knee joint for its superior analgesia, especially in settings where mild weakness of the thigh muscles would not interfere with postoperative rehabilitation. Early ambulation is not generally limited with the use of femoral block and the theoretical advantage of adductor canal block being "muscle sparring" was not observed in our study probably due to its suboptimal pain control.

\section{REFERENCES}

1. Duarte V, Fallis W, Slonowsky D et al. (2006): Effectiveness of femoral nerve blockade for pain control after total knee arthroplasty. J Perianesth Nurs., 21: 3116.

2. Memtsoudis S, Yoo D, Stundner O et al. (2015): Subsartorial adductor canal vs femoral nerve block for analgesia after total knee replacement. Int Orthop., 39: 673-80. 
3. Manickam B, Perlas A, Duggan E et al. (2009): Feasibility and efficacy of ultrasound guided block of the saphenous nerve in the adductor canal. Reg Anesth Pain Med., 34: 578-80.

4. Saranteas T, Anagnostis G, Paraskeuopoulos T et al. (2011): Anatomy and clinical implications of the ultrasound-guided subsartorial saphenous nerve block. Reg Anesth Pain Med., 36: 399-402.

5. Wang H, Boctor B, Verner J (2002): The effect of single injection femoral nerve block on rehabilitation and length of hospital stay after total knee replacement. Reg Anesth Pain Med., 27: 139-44.

6. Pelt C, Anderson A, Anderson M et al. (2014): Postoperative falls after total knee arthroplasty in patients with a femoral nerve catheter: can we reduce the incidence? J Arthroplasty, 29: 1154-1157.

7. Davis J, Bond T, Swenson J (2009): Adductor canal block: more than just the saphenous nerve? Reg Anesth Pain Med., 34: 618-619.

8. Sorensen J, Jæger P, Dahl J et al. (2016): The isolated effect of adductor canal block on quadriceps femoris muscle strength after total knee arthroplasty: a tripleblinded, randomized, placebo-controlled trial with individual patient analysis. Anesth Analg., 122: 553-8.

9. Koh J, Choi Y, Kim M et al. (2017): Femoral Nerve Block versus Adductor Canal Block for Analgesia after Total Knee Arthroplasty. Knee Surg Relat Res., 29 (2): 87-95.

10. Sessler C, Grap M, Ramsay M (2008): Evaluating and monitoring analgesia and sedation in the intensive care unit. Crit Care, 12 (3): 2-6.

11. Rahimzadeh P, Faiz H, Imani F et al. (2017): Relieving Pain after Arthroscopic Knee Surgery: Ultrasound-Guided Femoral Nerve Block or Adductor
Canal Block? Turkish Journal of Anaesthesiology and Reanimation, 45 (4): 218-224.

12. Kim D, Lin Y, Goytizolo E et al. (2014): Adductor canal block versus femoral nerve block for total knee arthroplasty: a prospective, randomized, controlled trial. Anesthesiology, 120: 540-50.

13. Wiesmann T, Piechowiak $\mathrm{K}$, Duderstadt $\mathrm{S}$ et al. (2016): Continuous adductor canal block versus continuous femoral nerve block after total knee arthroplasty for mobilisation capability and pain treatment: a randomised and blinded clinical trial. Arch Orthop Trauma Surg., 136: 397-406.

14. Elkassabany N, Antosh S, Ahmed M et al. (2016): The risk of falls after total knee arthroplasty with the use of a femoral nerve block versus an adductor canal block: a double-blinded randomized controlled study. Anesth Analg., 122: 1696-703.

15. Burckett-St Laurant $D$, Peng $P$, Giron Arango L et al. (2016): The nerves of the adductor canal and the innervation of the knee: an anatomic study. Reg Anesth Pain Med., 41: 321-7.

16. Ludwigson J, Tillmans $S$, Galgon $R$ et al. (2016): A Comparison of Single-Shot Adductor Canal Block vs Femoral Nerve Catheter for Total Knee Arthroplasty. J Arthroplasty, 31: 741-5.

17. Li D, Yang Z, Xie X et al. (2016): Adductor canal block provides better performance after total knee arthroplasty compared with femoral nerve block: a systematic review and meta-analysis. Int Orthop., 40: 925-33.

18. Machi A, Sztain J, Kormylo N et al. (2015): Discharge Readiness after Tricompartment Knee Arthroplasty: Adductor Canal versus Femoral Continuous Nerve Blocks-A Dual-center, Randomized Trial. Anesthesiology, 123: 444-456. 